\section{OPEN ACCESS}

Edited by:

Weien Yuan,

Shanghai Jiao Tong University, China

Reviewed by:

Paulo Cesar Morais,

Universidade

Católica de Brasilia, Brazil

Shi-Cong Tao,

Shanghai Sixth People's Hospital, China

${ }^{*}$ Correspondence:

Kari Murros

ext-murrokar@hus.fi

Johanna M. Salminen

johanna.m.salminen@helsinki.fi

Damien Faivre

damien.faivre@mpikg.mpg.de;

damien.faivre@cea.fr

Specialty section:

This article was submitted to Translational Medicine,

a section of the journal

Frontiers in Medicine

Received: 24 March 2019

Accepted: 16 May 2019

Published: 04 June 2019

Citation:

Murros K, Wasiljeff J,

Macías-Sánchez E, Faivre $D$

Soinne L, Valtonen J, Pohja M, Saari P,

Pesonen LJ and Salminen JM (2019)

Magnetic Nanoparticles in Human

Cervical Skin. Front. Med. 6:123.

doi: 10.3389/fmed.2019.00123

\title{
Magnetic Nanoparticles in Human Cervical Skin
}

\begin{abstract}
Kari Murros ${ }^{1 *}$, Joonas Wasiljeff ${ }^{2}$, Elena Macías-Sánchez ${ }^{3}$, Damien Faivre ${ }^{3,4 *}$, Lauri Soinne ${ }^{1}$, Jussi Valtonen ${ }^{5}$, Marjatta Pohja ${ }^{1}$, Pekka Saari ${ }^{6}$, Lauri J. Pesonen ${ }^{6}$ and Johanna M. Salminen ${ }^{2,6 *}$

1 Department of Neurology, Helsinki University Hospital, Helsinki, Finland, ${ }^{2}$ Department of Geosciences and Geography, University of Helsinki, Helsinki, Finland, ${ }^{3}$ Department of Biomaterials, Max Planck Institute of Colloids and Interfaces, Potsdam, Germany, ${ }^{4}$ CEA, CNRS, BIAM, Aix-Marseille University, Cadarache, France, ${ }^{5}$ Department of Plastic Surgery, Helsinki University Hospital, Helsinki, Finland, ${ }^{6}$ Department of Physics, University of Helsinki, Helsinki, Finland
\end{abstract}

Magnetic iron oxide nanoparticles, magnetite/maghemite, have been identified in human tissues, including the brain, meninges, heart, liver, and spleen. As these nanoparticles may play a role in the pathogenesis of neurodegenerative diseases, a pilot study explored the occurrence of these particles in the cervical (neck) skin of 10 patients with Parkinson's disease and 10 healthy controls. Magnetometry and transmission electron microscopy analyses revealed magnetite/maghemite nanoparticles in the skin samples of every study participant. Regarding magnetite/maghemite concentrations of the single-domain particles, no significant between-group difference was emerged. In low-temperature magnetic measurement, a magnetic anomaly at $\sim 50 \mathrm{~K}$ was evident mainly in the dermal samples of the Parkinson group. This anomaly was larger than the effect related to the magnetic ordering of molecular oxygen. The temperature range of the anomaly, and the size-range of magnetite/maghemite, both refute the idea of magnetic ordering of any iron phase other than magnetite. We propose that the explanation for the finding is interaction between clusters of superparamagnetic and single-domain-sized nanoparticles. The source and significance of these particles remains speculative.

Keywords: magnetite, nanoparticles, human tissue, skin, Parkinson's disease, superparamagnetic, gut

\section{INTRODUCTION}

Magnetic analyses of human tissues have revealed the presence of nanosized ferrimagnetic magnetite/maghemite particles (MNPs) in the brain, meninges, heart, liver, and spleen (1-7). The two distinct types of magnetic crystals identified in human brain are euhedral crystals possibly of biogenic origin $(1,5,8,9)$ and rounded crystals of combustion-derived origin (5). Many organisms are able to biochemically precipitate magnetite (10), and one theory is that biogenic magnetic nanoparticles in human tissues crystallize in situ, with the possible precursor being ferritin $(11,12)$.

The magnetic properties of magnetite depend on magnetic relaxation time (13), determined by particle size, morphology, the microscopic coercive force of the particle, saturation magnetization of the material, and thermal energy (14-17). Particles with ultrashort relaxation times are referred to as superparamagnetic (SPM). For magnetite, particles under $30 \mathrm{~nm}$ are considered SPM, whereas those of 30-80 nm are single-domain (SD) particles $(14,17,18)$. At room temperature, SD particles are able to carry magnetic remanence, while SPM are not (18). Generally, static magnetic fields can reduce mitochondrial membrane potentials, generate oxidative stress, and induce apoptotic processes $(19,20)$. Magnetic nanoparticles can translate possible effects to tissues. In human cells, 
magnetic nanoparticles have induced loss of mitochondrial membrane potential as an early sign of apoptosis (21). The magnetic iron oxide, magnetite $\left(\mathrm{Fe}^{2+} \mathrm{Fe}_{2}^{3+} \mathrm{O}_{4}\right)$, and its oxidized equivalent maghemite $\left(\gamma-\mathrm{Fe}_{2}^{3+} \mathrm{O}_{3}\right)$, may each possibly play a role in the pathogenesis of neurodegenerative diseases $(3,22)$. Iron and MNPs, by also containing ferric iron $\left(\mathrm{Fe}^{3+}\right)$, can promote alpha-synuclein protein ( $\alpha$-Syn) aggregation (23, 24). Importantly, the $\alpha$-Syn aggregates in the central and peripheral nerves are hallmarks of Parkinson's disease (PD) (25). Phosphorylated $\alpha$-Syn aggregates have been detectable in cervical skin samples of PD patients but not in control samples (26). Exploring the occurrence of MNPs in skin tissue may thus reveal important information on $\mathrm{PD}$ pathogenesis.

The primary aim of the present study was to examine the presence and potential role of MNPs in the cervical (neck) skin of subjects with and without PD. This site was targeted because it has most consistently harbored alpha-synuclein protein pathology in PD skin samples (26). We studied the mineralogy, morphology, and composition of MNPs in human cervical skin samples by magnetometry, transmission electron microscopy (TEM), and the associated spectroscopic techniques, electron energy loss spectroscopy (EELS) and energy-dispersive $\mathrm{X}$-ray spectroscopy (EDS).

\section{MATERIALS AND METHODS \\ Research Subjects and Ethical Issues}

The study participants formed two groups: 10 PD patients and a control group of 10 spouses of PD patients (Table 1). The patients had an idiopathic PD meeting the UK Parkinson's Disease Society Brain Bank clinical diagnostic criteria (27). None of the controls had PD nor any signs of parkinsonism. Exclusion criteria for both groups were cognitive decline (Mini-Mental State Examination points $<25$ ), bleeding disorders, anticoagulant treatment, diabetes, dermatological abnormalities in the cervical area, and allergy to local anesthetics. This prospective study was approved by the Ethics Committee of Helsinki and the Uusimaa Health District Area of Finland, and all procedures were in accordance with relevant guidelines and regulations. Each study subject provided a written informed consent.

TABLE 1 | Demographic and clinical characteristics of patients and controls.

\begin{tabular}{lcc}
\hline & Patients $(\boldsymbol{n}=\mathbf{1 0})$ & Controls $(\boldsymbol{n}=\mathbf{1 0})$ \\
\hline Age in years, median (range) & $68.5(55-74)$ & $70.5(50-72)$ \\
Gender, male (\%) & 50 & 50 \\
Years from PD diagnosis, median (range) & $8(1-17)$ & - \\
Hoehn \& Yahr stage, median (range) & $2.5(1.5-4.0)$ & - \\
Years with PD patient, median (range) & 0 & $43(1-50)$ \\
Body Mass Index, median (kg/m $\left.{ }^{2}\right)$ & 26.5 & 24.9 \\
Current smoking, prevalence (\%) & 10 & 30 \\
Hypertension (medicated), prevalence (\%) & 40 & 0 \\
Hyposmia by history, prevalence (\%) & 80 &
\end{tabular}

\section{Skin Samples}

Cervical (neck) skin tissue biopsies took place at the neurological outpatient clinic of the Helsinki University Central Hospital (HUCS), Jorvi Hospital. Each skin biopsy was performed by a plastic surgeon, who cut an approximately $10-\mathrm{mm}$ long boat-shaped section from the cervical skin area localized behind the right sternocleidomastoid muscle edge, anatomically corresponding to C7 spine level. The rims of the samples were removed with a knife having an aluminum shaft and a non-magnetic ceramic blade (Fine Science Tools, Heidelberg, Germany) to exclude possible contamination. Each isolated sample was packed into a non-magnetic polythene film package, and then kept in two sealed plastic bags in a freezer $\left(-20^{\circ} \mathrm{C}\right)$. To preclude skin-surface contamination, each skin sample, when frozen and dry, was macroscopically sectioned by the plastic surgeon with a ceramic blade into two parts: dermal (D) and epidermal (E). The D part was considered to contain some amounts of hypodermal tissue, whereas the epidermal part was a mixture of $\mathrm{E}$ and $\mathrm{D}$ tissue. The tissue samples remained in a polythene film package for room-temperature measurements and in plastic straw for low-temperature magnetic measurements. In one $\mathrm{PD}$ patient, the skin sample was too atrophic for proper dissection, excluding this case from the material of the present study.

\section{Magnetic Methods}

Room-temperature magnetic measurements took place at the Solid Earth Geophysics Laboratory, University of Helsinki, with 2G (now WSGI) cryogenic superconducting quantum interference device (SQUID) magnetometry. Each analytical step was designed and monitored to exclude any possible magnetic contamination. Instrument background noise level and level of laboratory contaminants were monitored with blank 5.8-g ice cubes of distilled deionized water in an ultrasonically cleaned plastic cube kept in the laboratory environment for 4-7 days before freezing. Typical ice-cube background noise levels were in the range of $6-9 \times 10^{-11} \mathrm{Am}^{2}$ (intensity: $1-1.6 \times 10^{-8}$ $\left.\mathrm{Am}^{2} \mathrm{~kg}^{-1}\right)$, corresponding with mean background noise level $\left(6-9 \times 10^{-11} \mathrm{Am}^{2}\right)$ of the instrument. To identify magnetic grain sizes and magnetic interactions, anhysteretic remanent magnetization (ARM) was induced in a decaying (from $100 \mathrm{mT}$ to zero) alternating magnetic field (af), with a small, superimposed direct current field $(0.05 \mathrm{mT})$, and was subsequently afdemagnetized. To study the type of magnetic material and its concentration, and to further study the grain size and magnetic interaction (28-30), we measured stepwise remanence acquisition with incremental application of direct current fields for subsets of samples (four patients, three controls), and room-temperature isothermal remanent magnetizations (IRMs). Saturation isothermal remanent magnetization (SIRM) was imparted with $3 \mathrm{~T}$ field and subsequently af-demagnetized using the same af-steps as in ARM demagnetization. The remanence of the sample holder was subtracted from the results.

Wohlfarth's ratio (W) $(28,29)$ was defined from the intersection of the IRM acquisition curve and the afdemagnetization curve of SIRM. For non-interacting SD particles 
with uniaxial anisotropy, Wohlfarth's ratio is 0.5 ; lower values are attributable to particle interactions, or to SPM or multidomain (MD) influences $(28,29)$. The Lowrie-Fuller test (30) served for study of the domain size of magnetic grains. To distinguish between SD- and MD-sized grains, requires comparison of the coercivity spectra of the ARM and IRM. In SD grains, ARM requires larger destructive fields than does IRM to reach the same normalized remanence level. The Lowrie-Fuller test can be quantized using the parameter MDF (median destructive field). MDF is calculated as the demagnetizing field required halving the magnetization.

Low temperature magnetic measurements of skin samples were done at the Low Temperature Laboratory, Aalto University (Espoo, Finland), using reciprocating sample SQUID Magnetic Property Measurement System XL7 magnetometer (Quantum Design) for the 10 patients and 10 controls. Care was taken that the airlock valve was operated properly to prevent any air leak into the system. For low temperature analyses a straw is used as a sample holder. After the sample was mounted into magnetometer, the sample space was purged with pure He a few times, and the measurements were done at very low He pressure in the measurement chamber. An oxygen test determined that there was no leak of air into the chamber. Induced magnetization was measured as a function of temperature between 5 and $270 \mathrm{~K}$ in a $50 \mathrm{mT}$ field after (1) the sample was cooled in a zero field (ZFC) and (2) cooled in an applied field of $50 \mathrm{mT}$ (FC). In addition, induced magnetization was measured as a function of the field for one patient sample up to $1 \mathrm{~T}$ in $48 \mathrm{~K}$ and in $300 \mathrm{~K}$. Measured magnetic moments were normalized with the masses of completely dry tissue samples (Tables 2, 3). Each completely dry mass was weighed after the low temperature measurement. For some of the dermal samples, dry mass was calculated based on the water content of the corresponding epidermal part.

\section{Magnetically Extracted Particles}

The magnetic extraction procedure was done for 11 dermal samples (nine patients and two controls). Extraction for the dermal sample was based on the procedure established by Hirt et al. (7) with slight modification. Briefly, the samples were first incubated for a day in a 1-mL solution consisting of $50 \mu \mathrm{L}$ of Proteinase $\mathrm{K}\left(5 \mathrm{mg} \mathrm{mL}^{-1}\right)+950 \mu \mathrm{L}$ HEPES Buffer $(50 \mathrm{mM})$. The samples were then centrifuged at $14,000 \mathrm{rpm}$ for $10 \mathrm{~min}$. The supernatant was discarded and the pellet was resuspended in a $1 \%$ Triton solution for another day before the preparation of TEM grids. After the initial trials, we decided to avoid magnetic separation, which failed to successfully separate magnetic particles from other nanoparticulate components.

\section{Transmission Electron Microscopy (TEM), Electron Energy Loss Spectroscopy (EELS) and Energy-Dispersive X-Ray Spectroscopy (EDS)}

TEM measurements took place in a double Cs-corrected Jeol JEM-ARM200F, equipped with a GIF Quantum Energy Filter
TABLE 2 | Magnetic properties of dermal samples.

\begin{tabular}{|c|c|c|c|c|c|}
\hline Sample & $\begin{array}{l}\text { Saturation } \\
\text { moment } \\
\left(10^{-10} \mathrm{Am}^{2}\right)\end{array}$ & $\begin{array}{l}\text { Dry } \\
\text { mass } \\
(\mathrm{mg})\end{array}$ & $\begin{array}{l}\text { Content of } \\
\text { SD magnetite } \\
(\mathrm{ng} / \mathrm{g})\end{array}$ & $\begin{array}{l}\text { Tp } \\
\text { (K) }\end{array}$ & $\begin{array}{l}\text { Tv } \\
\text { (K) }\end{array}$ \\
\hline \multicolumn{6}{|l|}{ PD PATIENTS } \\
\hline $1 \mathrm{D}$ & 6.22 & 8.0 & 1640.0 & & 20 \\
\hline $2 \mathrm{D}$ & 20.28 & 10.0 & 4408.7 & & 118 \\
\hline $3 D$ & 8.04 & 7.0 & 2428.1 & 41 & $121-123$ \\
\hline $6 \mathrm{D}$ & 51.23 & 4.0 & 27842.4 & & \\
\hline $8 \mathrm{D}$ & 6.00 & 11.0 & 1219.2 & 54 & 120 \\
\hline $10 D$ & 20.37 & 4.0 & 12652.2 & 48 & 121 \\
\hline $11 \mathrm{D}$ & 13.62 & 15.0 & 2028.0 & & \\
\hline $12 \mathrm{D}$ & 30.54 & 8.0 & 8852.2 & 46 & 118 \\
\hline $14 \mathrm{D}$ & 2.67 & 3.0 & 1733.0 & 42 & \\
\hline $18 \mathrm{D}$ & 6.43 & 15.0 & 904.4 & 54 & \\
\hline Mean & & & 6370.8 & & \\
\hline Average deviation & & & 6046.9 & & \\
\hline Median & & & 2228.1 & & \\
\hline \multicolumn{6}{|l|}{ CONTROLS } \\
\hline $5 \mathrm{D}$ & 8.15 & 8.0 & 2108.4 & & 121 \\
\hline $7 \mathrm{D}$ & 6.90 & 3.0 & 4713.3 & & 117 \\
\hline 9D & 3.56 & 8.0 & 943.0 & & \\
\hline $13 \mathrm{D}$ & 9.89 & 13.0 & 1653.8 & & \\
\hline $15 \mathrm{D}$ & 5.77 & 2.0 & 5700.6 & 42 & 123 \\
\hline $16 \mathrm{D}$ & 7.98 & 5.0 & 3854.1 & & 125 \\
\hline $17 \mathrm{D}$ & 16.11 & 20.0 & 1759.9 & & 115 \\
\hline $19 D$ & 11.01 & 13.0 & 1855.4 & & \\
\hline $20 D$ & 1.89 & 2.0 & 1786.4 & & \\
\hline $21 \mathrm{D}$ & 2.20 & 3.0 & 1913.0 & & \\
\hline Mean & & & 2628.8 & & \\
\hline Average deviation & & & 1276.3 & & \\
\hline Median & & & 1884.2 & & \\
\hline
\end{tabular}

$T p$, temperature for low temperature perturbation; $T v$, Temperature for Verwey transition.

(Gatan) and a silicon-drift energy-dispersive X-ray spectroscopy detector (Jeol). All spectroscopy measurements were done in STEM mode. EELS spectra were recorded using a dispersion of $0.1 \mathrm{eV} /$ channel, with a $5-\mathrm{mm}$ spectrometer entrance aperture, a convergence semi-angle $\alpha=20 \mathrm{mrads}$ and a collection semiangle $\beta=24$ mrads. The energy resolution was $1.2 \mathrm{eV}$ when measured at the full width at half maximum (FWHM) of the zero-loss peak. Due to rapid hydrocarbon deposition, only point measurements were chosen. Spectra presented here come from single particles, with an exposure time of $1 \mathrm{~s}$ and 60 frames summed (total integration time $60 \mathrm{~s}$ ); no smoothing was applied. Background was subtracted before each edge by use of powerlaw fitting, and plural scattering was removed by a Fourier-ratio deconvolution, both available in Digital Micrograph 3.1 software (Gatan Inc.). Plotting of the data and normalization were carried out with OriginPro 8.5 software. For energy-dispersive $\mathrm{X}$-ray spectroscopy, the specimen was tilted toward the detector $\left(\alpha\right.$-tilt $\left.=18^{\circ}\right)$ to increase detection efficiency. Acquisition and analysis of the data were completed with Analysis Station software (Jeol). 
TABLE 3 | Magnetic properties of epidermal samples.

\begin{tabular}{|c|c|c|c|c|c|}
\hline Sample & $\begin{array}{c}\text { Saturation } \\
\text { moment } \\
\left(10^{-10} \mathrm{Am}^{2}\right)\end{array}$ & $\begin{array}{c}\text { Dry } \\
\text { mass } \\
(\mathrm{mg})\end{array}$ & $\begin{array}{c}\text { Content of } \\
\text { SD magnetite } \\
\text { (ng/g) }\end{array}$ & $\begin{array}{l}\text { Tp } \\
\text { (K) }\end{array}$ & $\begin{array}{r}\text { Tv } \\
\text { (K) }\end{array}$ \\
\hline \multicolumn{6}{|l|}{ PD PATIENTS } \\
\hline $1 E$ & 10.84 & 13.9 & 1695.3 & & \\
\hline $2 E$ & 2.49 & 6.5 & 832.8 & & \\
\hline $3 E$ & 17.08 & 11.0 & 3375.5 & & \\
\hline $6 E$ & 8.68 & 8.3 & 2273.2 & & \\
\hline $8 \mathrm{E}$ & 21.31 & 7.5 & 6176.8 & & \\
\hline $10 \mathrm{E}$ & 11.70 & 11.2 & 2272.8 & & \\
\hline $11 E$ & 18.25 & 25.7 & 1543.7 & & \\
\hline $12 \mathrm{E}$ & 14.02 & 7.8 & 3907.5 & & \\
\hline $14 \mathrm{E}$ & 4.33 & 4.2 & 2243.3 & & \\
\hline $18 \mathrm{E}$ & 16.46 & 14.7 & 2434.2 & & 122 \\
\hline Mean & & & 2675.5 & & \\
\hline Average deviation & & & 1086.7 & & \\
\hline Median & & & 2273.0 & & \\
\hline \multicolumn{6}{|l|}{ CONTROLS } \\
\hline $5 E$ & 31.66 & 9.7 & 7095.5 & & \\
\hline $7 E$ & 3.87 & 4.5 & 1869.6 & & \\
\hline $9 \mathrm{E}$ & 6.18 & 6.1 & 2203.5 & & \\
\hline $13 \mathrm{E}$ & 11.69 & 14.9 & 1705.6 & & \\
\hline $15 E$ & 5.66 & 2.6 & 4729.1 & & \\
\hline $16 E$ & 9.94 & 4.6 & 4697.5 & & \\
\hline $17 \mathrm{E}$ & 16.11 & 18.7 & 1872.8 & 42 & 124 \\
\hline $19 E$ & 13.73 & 19.6 & 1522.8 & & \\
\hline $20 \mathrm{E}$ & 16.44 & 6.2 & 5764.4 & & \\
\hline $21 E$ & 5.48 & 10.7 & 1113.8 & & \\
\hline Mean & & & 3257.5 & & \\
\hline Average deviation & & & 1815.3 & & \\
\hline Median & & & 2038.2 & & \\
\hline
\end{tabular}

$T p$, temperature for low temperature perturbation; Tv, Temperature for Verwey transition.

\section{Statistics}

Study of statistical distributions of the measurements, as well as the between-group analysis with non-parametric comparison (Mann-Whitney U) and correlation (Spearman R) were carried out with Statistica 13.1 software (Dell Inc., Tulsa, OK, USA).

\section{RESULTS}

Alternating field (af) demagnetization of SIRM of the samples supports the presence of ferrimagnetic remanence carriers (Figures 1A,B). IRM acquisition curves showed that the skin tissue samples contained low-coercivity magnetic particles which were magnetically saturated by $300 \mathrm{mT}$, indicating ferrimagnetic magnetite/maghemite (Figures 1C-F). Wohlfarth's ratios $(28,29)$ between 0.19 and 0.24 for both PDs and controls were obtained (Figure 1). The MDF of SIRM for PD samples showed a larger range (7.9-20.5 $\mathrm{mT})$ than did those for control samples (10-18 $\mathrm{mT}$ ) (Figures 1, 2), indicating a wider range of domain size of remanence carriers for PD samples than for controls. The limited number of subjects did not, however, provide sufficient statistical power to detect a significant difference. The Lowrie-Fuller (30) test, where MDF values of ARM are higher than those of IRM, indicates that the SD size MNPs dominate over MD size in all of the samples.

The concentration of magnetic material in the samples was calculated, assuming that the material was magnetite, having saturation magnetization $92 \mathrm{Am}^{2} / \mathrm{kg}$ (1). The highest observed values of magnetite content were in the $\mathrm{PD}$ group, which displayed a higher spread of the distribution, most clearly in the dermal (D) samples (median $2228.1 \mathrm{ng} / \mathrm{g}$, range 904$27842 \mathrm{ng} / \mathrm{g}$; in controls, median $1884.2 \mathrm{ng} / \mathrm{g}$, range 943-5,700 ng/g) (Figure 2 and Table 2). Despite the trend, no significant difference appeared between the subpopulations $(p=0.34$, Mann-Whitney $U$-test). Values of the dermal samples showed no gender difference ( $p=0.12$, Mann-Whitney $U$-test) nor did they correlate with age or body mass index of study participants $(R=$ $-0.01, p=0.97$, and $R=-0.32, p=0.17$, respectively, Spearman Rank Correlation).

Induced magnetization, after both low-temperature experiments, where the sample was cooled in a zero field (ZFC) and cooled in an applied field of $50 \mathrm{mT}$ (FC), showed a rapid loss in magnetization on rewarming from 5 to $25 \mathrm{~K}$. This range includes the temperature range for a paramagnetic signal from blood in tissue $(2-8 \mathrm{~K})(7,31)$. With further warming, the ZFC showed an anomaly for the majority (six of ten) of the $\mathrm{D}$ samples of PD patients, and for one D sample and one E sample of the controls, with the peak ranging from 41 to $54 \mathrm{~K}$ (Tables 2, 3 and Figure 3). As to dermal samples, Fisher's exact test showed a trend-like difference in the occurrence of this anomaly between patients and controls $(p=0.06)$. The anomaly was absent for the FC curve. With continued warming, ZFC curves indicated Verwey transition for D samples of six $\mathrm{PD}$ patients at a temperature range of $118-123 \mathrm{~K}$ and for five $\mathrm{D}$ samples of controls at a temperature range of $115-125 \mathrm{~K}$ (Figure 3 and Table 2).

We measured induced magnetization as a function of the field for PD patient $18 \mathrm{D}$ near the low temperature anomaly peak at $48 \mathrm{~K}$ and at $300 \mathrm{~K}$ (Figure 4). After the diamagnetic correction, nearly saturated magnetization emerged at both temperatures, indicating ferrimagnetic material. A slightly higher coercivity value of $20 \mathrm{mT}$ appeared at $48 \mathrm{~K}$, compared to $15 \mathrm{mT}$ at $300 \mathrm{~K}$. At $48 \mathrm{~K}$, in the descending and ascending limbs of the curve in both positive and negative fields showed a bifurcation. At $300 \mathrm{~K}$, this behavior was absent.

TEM and EELS analyses of the extracted particles confirmed the occurrence of magnetite (Figure 5). The size of these particles varied in the range of $50-100 \mathrm{~nm}$, close to SD size, exceeding the size of nanoparticles formed within an 8 -nm diameter of the ferritin cors (10). The nanoparticles displayed a faceted morphology, in contrast to the rounded or spherical morphologies found in brain tissue and attributed to combustion-derived sources (5). Energy loss near ege structure (ELNES) analysis of the Fe L2,3-edge clearly shows a magnetite profile, both in patients and controls (Figure 5). The L3-edge presents a single maximum located at $709.4( \pm 0.1) \mathrm{eV}$, and L2-edge at 721-723 eV. The energy between the two lines being $13.2 \mathrm{eV}$ and the relative intensity 4.0, are in agreement 


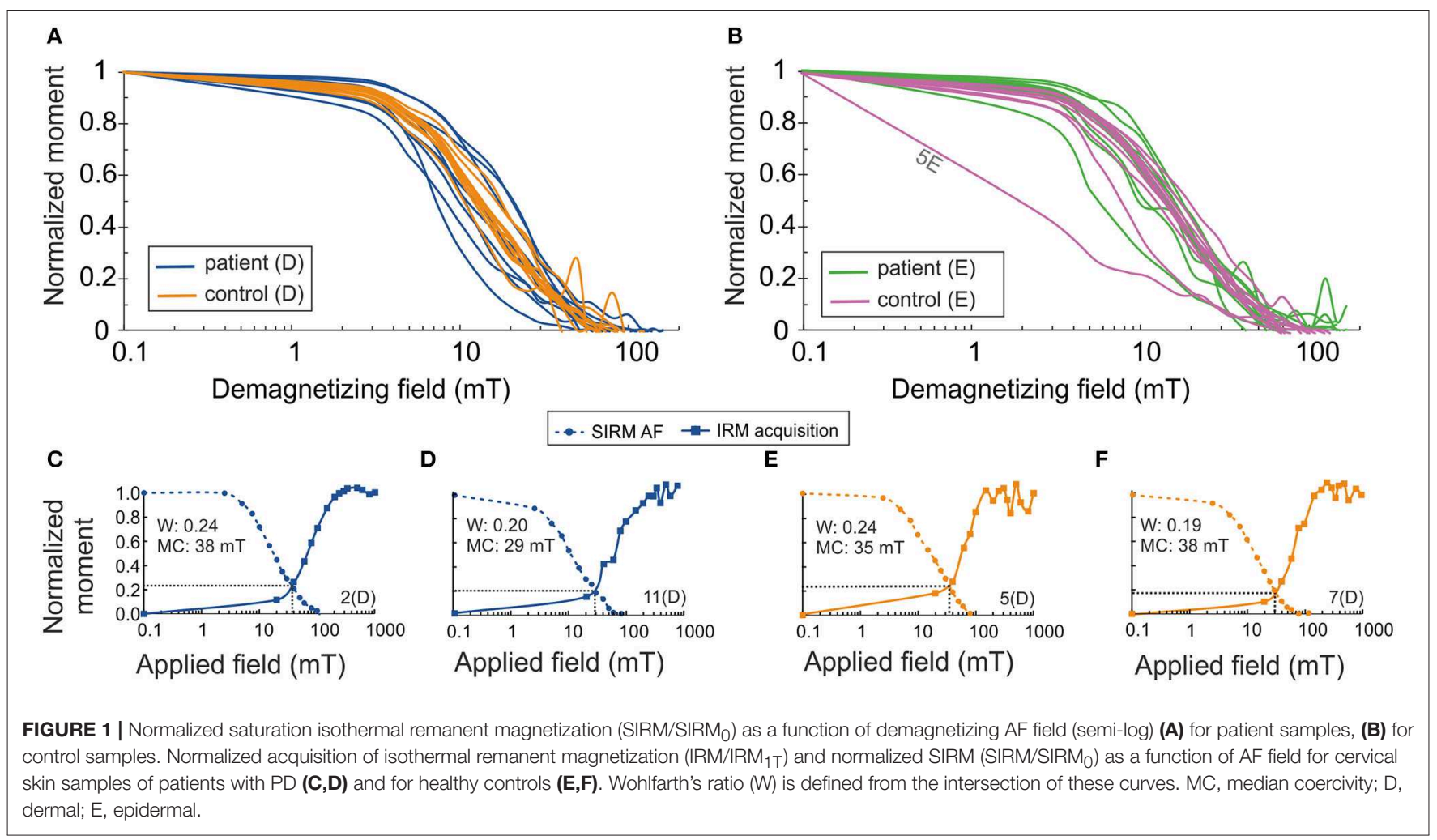

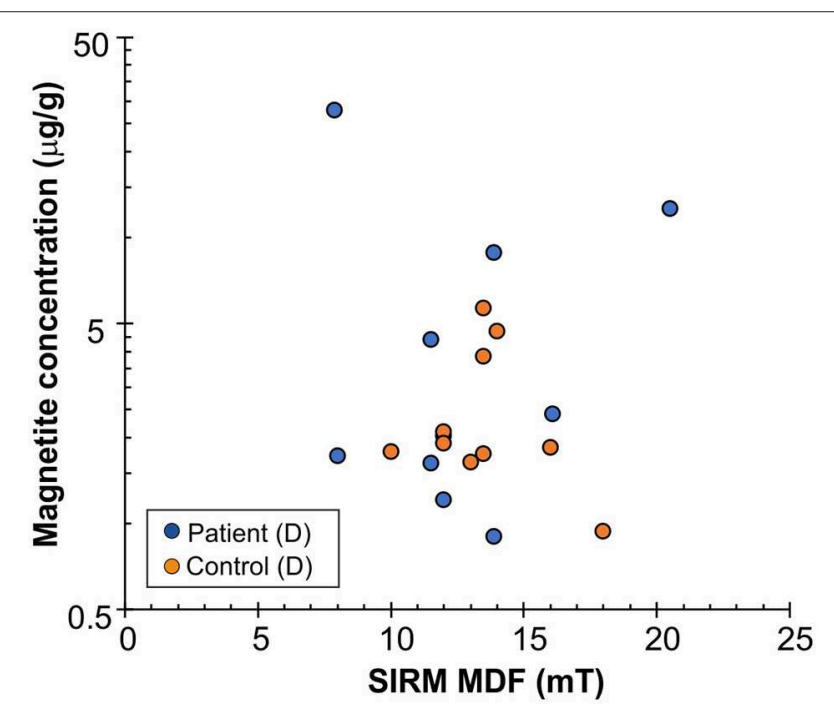

FIGURE 2 | Estimated magnetite concentration (micrograms per gram, $\mu \mathrm{g} / \mathrm{g}$ ) vs. median destructive field (MDF) of saturation isothermal remanent magnetization (SIRM) for dermal part of the cervical skin samples. Y-axis in logarithmic scale.

with earlier values for magnetite particles (32). The O K-edge shows a pre-peak around $530 \mathrm{eV}$ that presents an asymmetry, with a shoulder located at $530 \mathrm{eV}$ on the high-energy side, and a principal feature at $540 \mathrm{eV}$ that is common for all ironoxide phases.

\section{DISCUSSION}

Room-temperature IRM and SIRM measurements identified ferrimagnetic particles in all of the samples. The broad range of Verwey transition temperatures (PD: 118-123 K; controls: $115-125 \mathrm{~K})$ indicates both pure magnetite and partial oxidation of magnetite to maghemite or the presence of impurities (33), such as $\mathrm{Ti}^{4+}, \mathrm{Zn}^{2+}, \mathrm{Al}^{3+}$, in magnetite. TEM observations demonstrated that some of the particles were pure magnetite (Figure 5). Lowrie-Fuller test (30) refutes the presence of MD grains and Wohlfarth's ratios $<0.5$ for both PDs and controls indicate that the grains capable of carrying remanent magnetization at room temperature are magnetically interacting $(28,29)$. The median MNP concentrations for the dry skintissue samples were in the same range as reported for dry braintissue samples (5). Although the highest concentrations of MNPs came from the PD patients, no statistical difference between PD patients and controls emerged, possibly due to the small number of samples studied.

The majority of the $\mathrm{D}$ samples from $\mathrm{PD}$ patients showed anomalous behavior in the direct current magnetization at around $50 \mathrm{~K}$. Earlier low temperature measurements have indicated that heme-iron and ferritin do not contribute to the obtained anomaly around $50 \mathrm{~K}(7,31)$. The only iron phase in human tissue, one that has shown anomalous behavior in its magnetic properties around $50 \mathrm{~K}$, is magnetite/maghemite $(34,35)$. An anomaly at ca. $50 \mathrm{~K}$ in measurement of magnetic after-effect (MAE) or AC susceptibility has been associated with the onset of electron hopping upon warming and ionic 

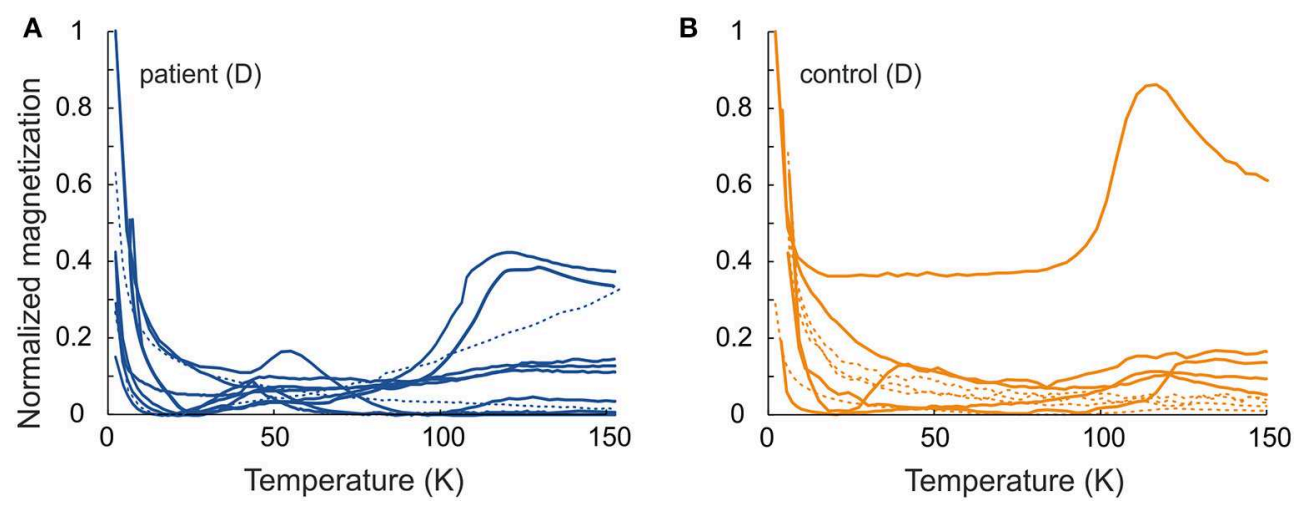

FIGURE 3 | Normalized induced magnetization as a function of temperature for ZFC $\left(\mathrm{M}_{\mathrm{ZfC}} / \mathrm{M}_{\mathrm{zfcmax}}\right)$ (A) patient samples and (B) control samples. Solid line-ca. $50 \mathrm{~K}$ anomaly or Verwey transition; dashed line-no low temperature features. For comparison, the magnetization values are normalized to maximum value of ZFC magnetization.

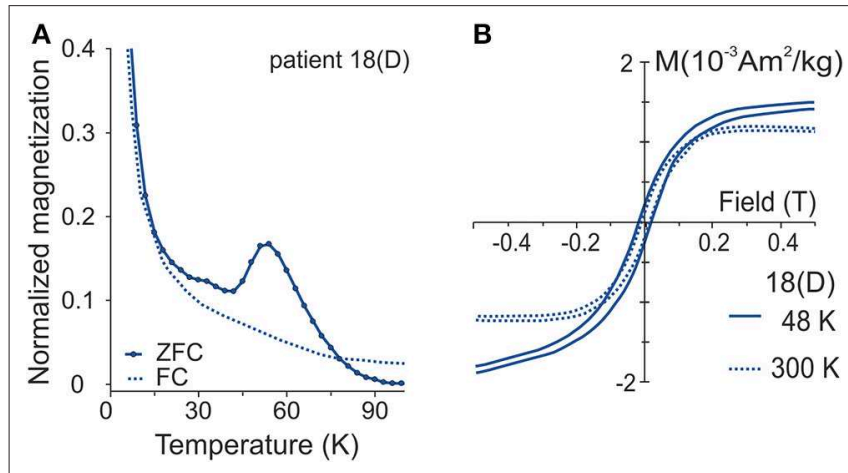

FIGURE 4 | (A) Normalized ZFC ( $\left.\mathrm{M}_{\mathrm{zfC}} / \mathrm{M}_{\mathrm{Zfcmax}}\right)$ and FC ( $\left.\mathrm{M}_{\mathrm{fC}} / \mathrm{M}_{\mathrm{fcmax}}\right)$ curves for patient sample 18D exhibiting $50 \mathrm{~K}$ perturbation. (B) Induced magnetization as a function of field near the anomaly at $48 \mathrm{~K}$ and in $300 \mathrm{~K}$ after slope correction. Normalization of ZFC-FC as in Figure $\mathbf{3}$.

ordering within domain walls, which indicate wall movement (36). However, the size of MNPs in this study indicates SD or SPM grain size, contradicting the presence of domain walls, being in line with the size range of MNPs in human brain tissue (7).

In 1998, Moskowitz et al. associated a low-temperature anomaly during ZFC analyses with SPM- to SD-sized maghemite particles (partly aggregated clusters) in magnetoferritin (37). Accordingly, we propose that the $\sim 50 \mathrm{~K}$ anomaly indicates magnetically interacting clusters of possible SPM- and SD- sized ferrimagnetic MNPs. The peak for this perturbation occurred only on the ZFC curve, since, due to increased temperature, initially SD sized particles progressively unblock, align their moments with the applied field, and eventually randomize due to thermal energy. This anomaly did not occur during the FC analyses, since magnetization was already aligned with the field during the cooling through blocking temperature and this experiment only shows the demagnetization of the blocked particles.

Additional support for SPM grains comes from low temperature hysteresis measurements, which show higher coercivity at $48 \mathrm{~K}$ than at room temperature (Figure 4B), indicating the presence of SPM grains as being able to carry remanence at $48 \mathrm{~K}$ (4). The bifurcations similar to those obtained in the hysteresis loop have been evident in studies on human tissue $(7,36)$, with a suggestion that the switching of the ab crystalline axes of magnetite around $45 \mathrm{~K}$ could possibly explain this bifurcation (36). In the initial field, the easy axis of magnetization would lie along one of the axes. As the field is reduced and then raised to the starting value again, what may occur is a switch in preferred axis, leading to the bifurcation observed.

Another explanation for the anomaly at $\sim 50 \mathrm{~K}$ may be the magnetic ordering of molecular oxygen, which undergoes an antiferromagnetic transition at about $43 \mathrm{~K}$ being strongly paramagnetic above this temperature (38). We took care to prevent any oxygen leak into the system. Furthermore, the magnetic intensity of the $\sim 50 \mathrm{~K}$ signal was for most of the samples an order of magnitude larger $\left(10^{-4} \mathrm{Am}^{2} / \mathrm{kg}\right)$ than known effects related to the magnetic ordering of oxygen at $43 \mathrm{~K}(<2$ $\times 10^{-5} \mathrm{Am}^{2} / \mathrm{kg}$ ) (7), providing additional evidence against the oxygen as a cause for the $\sim 50 \mathrm{~K}$ anomaly.

Our magnetometric studies revealed MNPs with SD characteristics in all skin samples and interacting SPM characteristics for the majority of the $\mathrm{D}$ samples from the PD patients. The shapes of the SD-size magnetic nanoparticles studied by TEM did not resemble the cubo-octahedral crystals of possible biogenic magnetite $(1,9)$, but merely resembled non-biogenic magnetite. No rounded or spherical morphologies were detectable. EEL spectra of four samples demonstrated that their particles were composed of magnetite (Figure 5); on the basis of their size, we propose a source different from that of ferritin cores.

The origin of MNPs in human tissues has remained speculative (biogenic, non-biogenic, or anthropogenic/combustion). Polluted air (combustion-derived MNPs) via the respiratory route has been proposed as one source of these MNPs (5). The olfactory route was considered to be the passageway to the brain for these round and spherical particles (5), but was recently contradicted (6). Here, no particles were detectable with such morphology. However, we cannot 

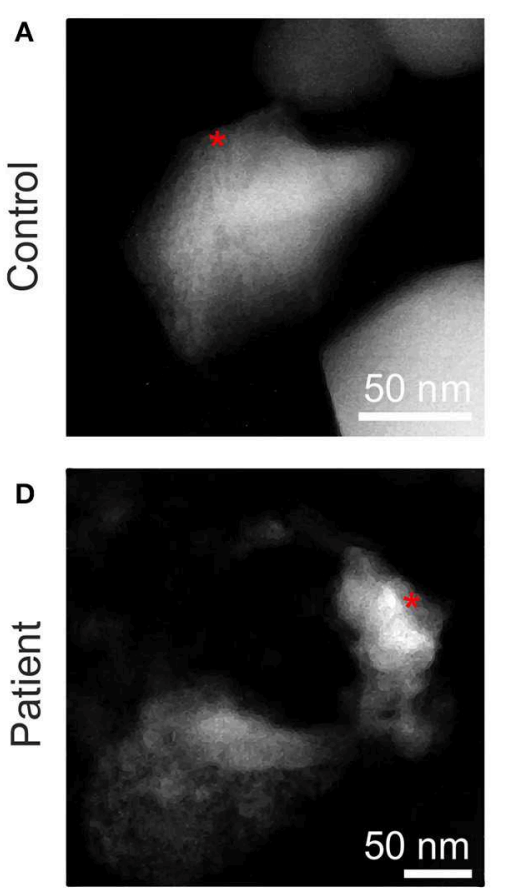

B

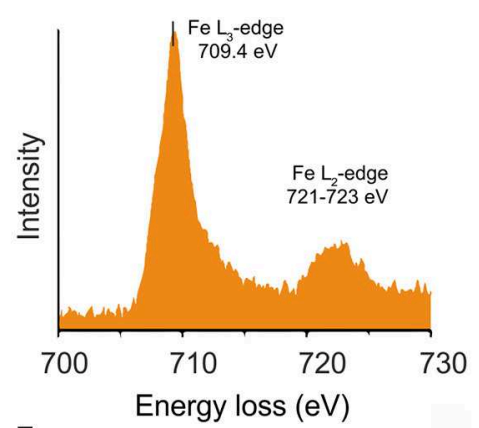

E

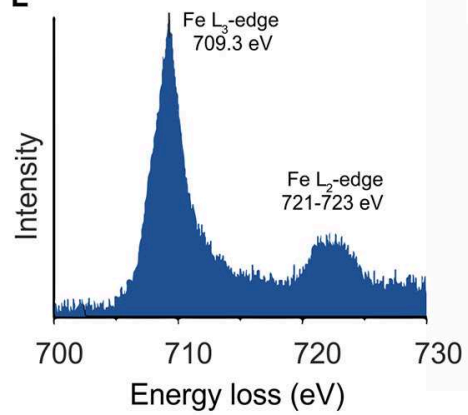

C

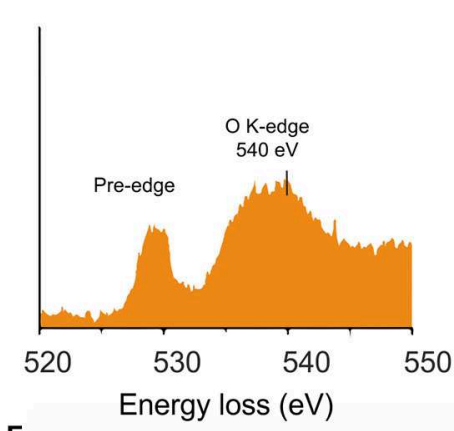

$\mathbf{F}$

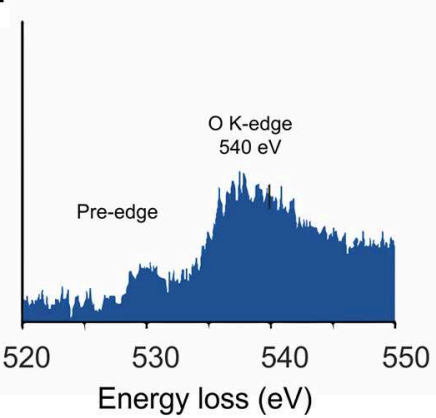

FIGURE 5 | High annular dark field (HAADF) image of particles extracted from human cervical skin with respective EEL spectra; A-C (Healthy control), D-F (PD patient). Iron $\mathrm{L}_{2.3}$-edge $(\mathbf{B}, \mathbf{E})$ and oxygen $\mathrm{K}$-edge fine structure $(\mathbf{C}, \mathbf{F})$ confirm that particles are composed of magnetite.

fully exclude that airborne MNPs, via a respiratory route or even through skin structures, may in part explain the results. Low-temperature magnetic measurements showed the presence of apparent SPM particles mainly in the dermal samples of PD patients (Tables 2, 3), hinting strongly of a source other than the skin surface. As to intrinsic sources, the ferrihydrite core of the iron-storage protein ferritin has been proposed to be a precursor of MNPs $(11,12)$. Considerable argumentation goes against this view, as the size of single-domain MNPs is not compatible with the 6- to 8-nm diameter of the inner cavity of the ferritin protein (10). Further, nuclear magnetic resonance relaxometry studies have shown that the content of magnetite in the ferrihydrite core is $<1 \%$ (39). At least, what is not ruled out is that ferritin could by some mechanism produce small superparamagnetic MNPs, which can then aggregate into larger MNPs with SD-like characteristics. Experimens have shown that nucleation and growth of magnetite proceeds through rapid agglomeration of nanosized primary particles (40). Recently, it has been reported that human stem cells have an ability to synthesize magnetic nanoparticles from nanodegeneration products (41).

A potential source of MNPs may be the gastrointestinal tract. Having been present in many organisms (10), MNPs may be expected to be found in various foodstuffs, which, when ingested, are probably absorbed into the bloodstream from the gastrointestinal tract. In addition, drinking-water can contain MNPs (42). Ferrihydrite in soils deserves attention, since in its amorphous form, it can percolate into groundwaters and further into drinking-water (43), and magnetite crystal formation may occur by an abiotic mechanism (44). As to bacteria sporadically residing in the human gastrointestinal tract, sulfate-reducing Desulfovibrio species are able to synthesize magnetite (4547). As these bacteria also produce hydrogen sulfide, known to cause olfactory dysfunction (48), a prevalent phenomenon in PD (49), the potential occurrence of these bacteria in PD is relevant. The uptake of nanoparticles from the intestinal lumen into the blood circulation may take place mainly through an endocytic transcellular transport, with even 100-nm-sized particles transported (50). Iron nanoparticles can evidently cross plasma membranes by a non-endocytic pathway, gaining direct access to the cytoplasm (51). The gut epithelial enteroendocrine cells, known to contain $\alpha$-Syn, may therefore be structures to display $\alpha$-Syn aggregation promoted by magnetite nanoparticles $(24,52,53)$. In the blood, nanoparticles become coated by proteins, small molecules and ions (50). Tissue macrophages are considered the primary cell types that phagocytose nanoparticles which have ended up in the blood circulation (54). As the macrophage density in normal human dermis is considerable (55), skin may play an important role in removing MNPs from the blood circulation. This view is supported by an animal study showing intravenously administered nanoparticles to accumulate in the dermis, first localizing in dermal macrophages (56). The substantial magnetite content of the skin samples detected in this study may reflect such a process.

MNPs can evidently interfere with cell functions. Interactions of SPM particles with prolonged external magnetic fields may lead to biological impacts, because SPM particles have a 
strong magnetic susceptibility; relatively weak static magnetic fields can induce loss of membrane potentials in mitochondria $(19,20)$, which may potentially lead to mitophagy (56). Magnetite nanoparticles per se may induce mitochondrial dysfunction (21), and promote, in an uncoated form, $\alpha$-Syn aggregation (24).

In the present pilot study, the number of samples was too small to allow definitive conclusions as to the true differences in MNP characteristics and quantities between the study groups. As an additional factor, PD is a disease with diverse characteristics in which various pathophysiological mechanisms may come into play (57), and the overall accuracy of clinical diagnosis of PD is far from satisfactory particularly in its early stages $(58,59)$. Notably, relatively high amounts of singledomain MNPs were observable in all cervical skin samples. A possible clustering of such particles with superparamagnetic particles needs further exploration in Parkinson's disease. Our study raises questions as to the origin and role of MNPs in physiologic and pathologic states in human beings as well as to the importance of the skin as an internalizer of these particles.

\section{DATA AVAILABILITY}

The datasets generated for this study are available on request to the corresponding author JS.

\section{ETHICS STATEMENT}

Ethics Committee of Helsinki and Uusimaa Health District Area of Finland.

\section{AUTHOR CONTRIBUTIONS}

$\mathrm{KM}$ and JS contributed equally to the work in this research. KM, JS, LP, DF, and EM-S designed the research. KM conceived the original idea. KM, JS, JW, JV, DF, EM-S, LS, MP, and PS performed research. JS, KM, JW, LS, LP, DF, and EM-S analyzed the data. KM, JS, and JW wrote the manuscript, including input from EM-S, DF, LS, and LP.

\section{REFERENCES}

1. Kirschvink JL, Kobayashi-Kirschvink A, Woodford BJ. Magnetite biomineralization in the human brain. Proc Natl Acad Sci USA. (1992) 89:7683-7. doi: 10.1073/pnas.89.16.7683

2. Dobson J, Grassi P. Magnetic properties of human hippocampal tissueevaluation of artefact and contamination sources. Brain Res Bull. (1996) 39:255-9. doi: 10.1016/0361-9230(95)02132-9

3. Pankhurst Q, Hautot D, Khan N, Dobson J. Increased levels of magnetic compounds in Alzheimer's disease. J Alzheimers Dis. (2008) 13:49-52. doi: 10.3233/JAD-2008-13105

4. Grassi-Schultheiss PP, Heller F, Dobson J. Analysis of magnetic mineral in the human heart, spleen and liver. Biometals. (1997) 10:351-35. doi: 10.1023/A:1018340920329

\section{CONTRIBUTION TO THE FIELD}

Human internal organs have been reported to harbor magnetic nanoparticles. In 1992, Kirschvink and his colleagues were the first to report magnetite nanoparticles in human brain tissue. Later, apparent magnetite and maghemite particles were found in the human heart, spleen, and liver. With their magnetic properties, these iron oxide particles may have the propensity to induce deleterious effects in cells and play a role in the pathogenesis of neurodegenerative diseases. The present study, using magnetometric and electron microcopy methodology, revealed ferrimagnetic single-domainsized magnetite/maghemite nanoparticles in all skin samples of patients with Parkinson's disease as well as in those of healthy controls, and revealed superparamagnetic material in several samples. These findings complement studies focused on magnetic material in human tissues. The particles are proposed to be airborne or gut-mediated, and the skin may play a role in internalizing them from the blood circulation. The present findings open a new perspective on the occurrence of magnetic nanoparticles in human tissues and underline the need to clarify not only the origin of these particles but also their role in physiologic and pathologic states. To this end, the skin offers a convenient target.

\section{FUNDING}

For financial support the authors thank Helsinki University Central Hospital and the Finnish Parkinson Foundation. JS was funded by the Academy of Finland. DF acknowledges support from the Max Planck Society.

\section{ACKNOWLEDGMENTS}

The authors thank Dr. Alexander Savin (Aalto University, Helsinki) for his technical help with MPMS measurements, Prof. Sauli Savolainen (Helsinki University) for his help with recruiting MSc. student Pekka Saari to the study team, Dr. Marge Kartau and Dr. Tua Annanmäki (Helsinki University Central Hospital, HUCS) for their help in clinical matters, Dr. Anna Murros for her comments on the manuscript, and nurses Marianne Ikola, Pirjo Nurmi and Sari Karesvuori (HUCS) for their assistance in biopsy procedures.

5. Maher BA, Ahmed IAM, Karloukovski V, MacLaren DA, Foulds PG, Allsop D, et al. Magnetite pollution nanoparticles in the human brain. Proc Natl Acad Sci USA. (2016) 113:10797-1801. doi: 10.1073/pnas.16059 41113

6. Gilder SA, Wack M, Kaub L, Roud SC, Petersen N, Heinsen H, et al. Distribution of magnetic remanence carriers in the human brain. Sci Rep. (2018) 8:11363. doi: 10.1038/s41598-018-29766-z

7. Hirt AM, Brem F, Hanzlik M, Faivre D. Anomalous magnetic properties of brain tissue at low temperature: the $50 \mathrm{~K}$ anomaly. J Geophys Res. (2006) 111:B12S06. doi: 10.1029/2006JB004570

8. Schultheiss-Grassi PP, Wessiken R, Dobson J. TEM investigations of biogenic magnetite extracted from the human hippocampus. Biochim Biophys Acta. (1999) 1426:212-6. doi: 10.1016/S0304-4165(98) 00160-3 
9. Kirschvink J, Kobayashi-Kirschvink A, Diaz-Ricci JC, Kirscvink SJ. Magnetite in human tissues: a mechanism for the biological effects of weak ELF magnetic fields. Bioelectromag Suppl. (1992) 1:101-13. doi: 10.1002/bem.2250130710

10. Gorobets O, Gorobets S, Koralewski M. Physiological origin of biogenic magnetite nanoparticles in health and disease: from bacteria to humans. Int J Nanomed. (2017) 12:4371-95. doi: 10.2147/IJN.S130565

11. Kobayashi A, Yamamoto N, Kirschvink J. Study of inorganic crystalline solids in biosystems: magnetite in the human body. J Japan Soc Powder and Powder Metall. (1996) 43:1354-60. doi: 10.2497/jjspm.43.1354

12. Quintana C, Gutiérrez L. Could a dysfunction of ferritin be a determinant factor in the aetiology of some neurodegenerative diseases? Biochim Biophys Acta. (2010) 1800:770-82. doi: 10.1016/j.bbagen.2010.04.012

13. Néel L. Théorie du traînage magnétique des ferromagnétiques en grains finns avec application aux terres cuites. Annales Geophysicae. (1949) 5:99-136.

14. Reichel V, Kovács A, Kumari M, Bereczk-Tompa E, Schneck E, Diehle P, et. al. Single crystalline superstructured stable single domain magnetite nanoparticles. Sci Rep. (2017) 7:45484. doi: 10.1038/srep45484

15. Muxworthy AR, Williams L. Critical single-domain/multidomain grain sizes in noninteracting and interacting elongated magnetie particles: implications for magnetosomes. J Geophys Res. (2006) 111:B12S12. doi: 10.1029/2006JB004588

16. Muxworthy AR, Williams W. Critical superparamagnetic/singledomain/multidomain grain sizes in noninteracting and interacting elongated magnetie particles: implications for magnetosomes. J Royal Soc Interface. (2008) 6:1207-12. doi: 10.1098/rsif.2008.0462

17. Dunlop DJ. Superparamagnetic and single-domain threshold sizes in magnetite. J Geophys Res. (1973) 78:1780-1179. doi: 10.1029/JB078i011p01780

18. Newell AJ, Merrill RT. Single-domain critical sizes for coercivity and remanence. J Geophys Res. (1999) 104:617-62. doi: 10.1029/1998JB90 0039

19. Calabrò E, Condello S, Currò M, Ferlazzo N. Effects of low intensity static magnetic field on FTIR spectra and ROS production in SH-SY5Y neuronal-like cells. Bioelectromagnetics. (2013) 34:618-29. doi: 10.1002/bem. 21815

20. Raja SO, Dasgupta AK. Instant response of live HeLA cells to static magnetic field and its magnetic adaptation. Cell Behav. (2014) arXiv:1407.3499v1.

21. Zhu MT, Wang Y, Feng WY, Wang B, Ouyang H, Chai ZF. Oxidative stress and apoptosis induced by iron oxide nanoparticles in cultures umbilical endothelial cells. J Nanosci Nanotechnol. (2010) 10:8584-90. doi: $10.1166 /$ jnn. 2010.2488

22. Gieré R. Magnetite in the human body: Biogenic vs. anthropogenic. Proc Natl Acad Sci. (2016) 113:11986-7. doi: 10.1073/pnas.1613349113

23. Carboni E, Lingor P. Insights on the interaction of alpha-synuclein and metals in the pathophysiology of Parkinson's disease. Metallomics. (2015) 7:395-404. doi: 10.1039/C4MT00339J

24. Joshi N, Basak S, Kundu S, De G, Mukhopadhyay A, Chattopadhyay K. Attenuation of the early events of $\alpha$-synuclein aggregation: a fluorescence correlation spectroscopy and laser scanning microscopy study in the presence of surface-coated $\mathrm{Fe}_{3} \mathrm{O}_{4}$ nanoparticles. Langmuir. (2015) 31:146978. doi: 10.1021/la503749e

25. Del Tredici K, Braak H. Sporadic Parkinson's disease: development and distribution of $\alpha$-synuclein pathology. Neuropathol Appl Neurobiol. (2016) 42:33-50. doi: 10.1111/nan.12298

26. DonadioV, Incensi A, Leta V, Giannoccaro MD, Scaglione C, Martinelli P, et al. Skin nerve $\alpha$-synuclein deposits: a biomarker for idiopathic Parkinson disease. Neurology. (2014) 82:1362-9. doi: 10.1212/WNL.0000000000000316

27. Massano J, Bhatia, KP. Clinical approach to Parkinson's disease: features, diagnosis, and principles of management. Cold Spring Har. Perspect Med. (2012) 2:a008870. doi: 10.1101/cshperspect.a008870

28. Wohlfarth EP. Relations between different modes of acquisition of the remanent magnetization of ferromagnetic particles. J Appl Phys. (1958) 29:595. doi: $10.1063 / 1.1723232$

29. Cisowski S. Interacting vs. non-interacting single-domain behavior in natural and synthetic samples. Phys Earth Planet Inter. (1981) 26:77-83. doi: 10.1016/0031-9201(81)90097-2

30. Lowrie W, Fuller M. On the alternating field demagnetisation characteristics of multi-domain thermoremanent magnetization in magnetite. J Geophys Res. (1971) 76:6339-49. doi: 10.1029/JB076i026p06339
31. Brem F, Hiert AM, Winklhofer M, Frei K, Yonekawa Y, Wieser H, et al. Magnetic iron compounds in the human brain: a comparison of tumour and hippocampal tissue. J Royal Soc Interphase. (2006) 3: 833-841. doi: 10.1098/rsif.2006.0133

32. Colliex C, Manoubi T, Oritz C. Electron energy loss spectroscopy near edge fine structures in the iron-oxygen system. Phys Rev B. (1991) 44:11402-11. doi: 10.1103/PhysRevB.44.11402

33. Özdemir Ö, Dunlop DJ. Hallmarks of maghemitization in low-temperature remanence cycling of partially oxidized magnetite nanoparticles. J Geophys Res. (2010) 115:B02101. doi: 10.1029/2009JB006756

34. Walz F, Kronmüller $H$. Evidence for a single-stage Verweytransition in perfect magnetite. Philos Mag B. (1991) 84:623-8. doi: $10.1080 / 13642819108217886$

35. Moskowitz BM, Jackson MJ, Kissel C. Low-temperature magnetic behavior of titanomagnetites. Earth Planet Sci Lett. (1998) 157:141-9. doi: 10.1016/S0012-821X(98)00033-8

36. Inase T, Miyamoto Y. Anomalous behavior in temperature dependence of pyroelectric polarization and magnetoelectric polarization of magnetite below 60 K. J. Phys Soc Jpn. (1987) 56:3683-8. doi: 10.1143/JPSJ.56.3683

37. Moskowitz BM, Frankel RB, Walton SA, Dickson DPE, Wang KKW, Douglas $\mathrm{T}$, et al. Determination of the pre-exponential frequency factor for superparamagnetic maghemite particles in magnetoferritin. J Geophys Res. (1997) 102:22671-80. doi: 10.1029/97JB01698

38. Schubert S. Magnetic susceptibility of oxygen adsorbed on graphite. Phys Rev Lett. (1978) 40:723-5. doi: 10.1103/PhysRevLett.40.723

39. Gossuin Y, Hautot D, Muller RN, Pankhurst Q, Dobson J, Morris C, et al. Looking for biogenic magnetite in brain ferritin using NMR relaxometry. NMR Biomed. (2005) 18:469-72. doi: 10.1002/nbm.983

40. Baumgartner J, Dey A, Bomans PH, Le Coadeu C, Fratzl P, Sommerdijk NA, et al. Nucleation and growth of magnetite solution. Nat Mater. (2013). 12:310-4. doi: 10.1038/nmat3558

41. Van de Walle A, Sangnier AP, Abou-Hassan A, Curcio A, Hémadi M, Menguy $\mathrm{N}$, et al. Biosynthesis of magnetite nanoparticles from nanodegeneration products revealed in human stem cells. Proc Natl Acad Sci USA. (2019) 116:4044-53. doi: 10.1073/pnas.1816792116

42. Barkatt A, Pulvirenti AL, Adel Hadadi MA, Viragh C. Composition and particle size of superparamagnetic corrosion products in tap water. Water Res. (2009) 43:3319-25. doi: 10.1016/j.watres.2009.04.048

43. Vodyanitskii YN, Shoba SA. Ferrihydrite in soils. Eur J Soil Sci. (2016) 49:796-80. doi: 10.1134/S1064229316070127

44. Hansel C, Brenner SG, Neiss J, Dohnalkova A. Secondary mineralization pathways induced by dissimilatory iron reduction of ferrihydrite under advective flow. Geochim Cosmochim Acta. (2003) 67:2977-92. doi: 10.1016/S0016-7037(03)00276-X

45. Goldstein EJC, Citro DM, Peraino VA, Cross SA. Desulfovibrio desulfuricans bacteremia and review of human desulfovibrio infections. J Clin Microbiol. (2003) 41:2752-4. doi: 10.1128/JCM.41.6.2752-27 54.2003

46. Chistyakova NI, Rusakov VS, Zavarzina DG, Slobodkin AI, Gorohova TV. Mössbauer study of magnetite formation by iron- and sulfatereducing bacteria. Hyperfine Interact. (2004) 156/157: 411-415. doi: 10.1023/B:HYPE.0000043261.17920.b9

47. Lovley DR, Roden EE, Phillips EJP, Woodward J. Enzymatic iron and uranium reduction by sulfate-reducing bacteria. Mar Geol. (1993) 113:41-53. doi: 10.1016/0025-3227(93)90148-O

48. Hirsch AR, Zavala G. Long term effects on the olfactory system of exposure to hydrogen sulphide. Occup Environ Med. (1999) 56:284-7. doi: 10.1136/oem.56.4.284

49. Doty RL. Olfaction in Parkinson's disease and related disorders. Neurobiol Dis. (2012) 46: 527-55. doi: 10.1016/j.nbd.2011.10.026

50. Bergin LL, Witzmann FA. Nanoparticle toxicity by the gastrointestinal route: evidence and knowledge gaps. Int J Biomed Nanosci Nanotechnol. (2013) 3:1-2. doi: 10.1504/IJBNN.2013.054515

51. Zanella D, Bossi E, Gomati R, Bastos C, Faria N, Bernardini G. Iron oxide nano-particles can cross plasma membranes. Sci. Rep. (2017) 7:11413. doi: 10.1038/s41598-017-11535-Z

52. Chandra R, Hiniker A, Kuo Y, Nussbaum RL, Liddle RA. $\alpha$-Synuclein in gut endocrine cells and its implications for Parkinson's disease. J Clin Invest. (2017) 2:e92295 doi: 10.1172/jci.insight.92295 
53. Liddle RA. Parkinson's disease from the gut. Brain Res. (2018) 15:201-6. doi: 10.1016/j.brainres.2018.01.010

54. Gustafson HH, Holt-Casper D, Graniger DW, Ghandehari H. Nanoparticle uptake: the phagocyte problem. Nano Today. (2015) 10:487-510. doi: 10.1016/j.nantod.2015.06.006

55. Weber-Matthiesen K, Sterry W. Organization of the monocyte/macrophage system of normal human skin. J Invest Dermatol. (1990) 95:83-9. doi: 10.1111/1523-1747.ep12874002

56. Sykes EA, Dai Q, Tsoi KM, Hwang DM, Chan WCW. Nanoparticle exposure in animals can be visualized in the skin and analyzed via skin biopsy. Nat Commun. (2014) 13:3796 doi: 10.1038/ ncomms 4796

57. Ding WX, Yin XM. Mitophagy: mechanims, pathophysiological roles, and analysis. Biol Chem. (2012) 393:547-64. doi: 10.1515/hsz-2012-0119

58. Lees JA. Parkinson chimaera. Neurology. (2009) 72(Suppl. 2):S2-11. doi: 10.1212/WNL.0b013e318198daec
59. Rizzo G, Copetti M, Arcuti S, Martino D, Fontana A, Logroscino G. Accuracy of clinical diagnosis of Parkinson disease: a systematic review and metaanalysis. Neurology. (2016) 86:566-76. doi: 10.1212/WNL.0000000000002350

Conflict of Interest Statement: The authors declare that the research was conducted in the absence of any commercial or financial relationships that could be construed as a potential conflict of interest.

Copyright (c) 2019 Murros, Wasiljeff, Macías-Sánchez, Faivre, Soinne, Valtonen, Pohja, Saari, Pesonen and Salminen. This is an open-access article distributed under the terms of the Creative Commons Attribution License (CC BY). The use, distribution or reproduction in other forums is permitted, provided the original author(s) and the copyright owner(s) are credited and that the original publication in this journal is cited, in accordance with accepted academic practice. No use, distribution or reproduction is permitted which does not comply with these terms. 